IFUP-TH 12/94

\title{
The SU(3) deconfining phase transition with Symanzik action
}

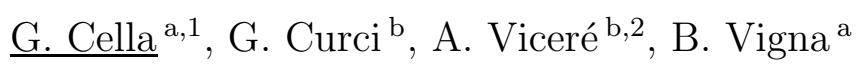 \\ a Dipartimento di Fisica dell'Università di Pisa, Piazza Torricelli 2, I-56126 \\ Pisa, ITALY, e-mail cella@sun10.difi.unipi.it \\ b Istituto Nazionale di Fisica Nucleare, Piazza Torricelli 2, I-56126 Pisa, \\ ITALY, e-mail vicere@sun10.difi.unipi.it
}

\begin{abstract}
We report on the determination of the deconfining temperature in $S U(3)$ pure gauge theory, using the Symanzik tree level improved action, on lattices of size $3 \times 12^{3}, 4 \times 16^{3}, 5 \times 20^{3}, 6 \times 24^{3}$. We find that the asymptotic scaling violation pattern is similar to the one observed using the Wilson action. We conclude that the irrelevant operators do not affect, in the range of couplings considered, the lattice $\beta$ function. An analysis based on an effective coupling formulation shows an apparent improvement.
\end{abstract}

\section{Introduction}

In an earlier work [1] we have reported on a high statistics investigation of the effect of the Symanzik tree improvement [2], based on the study of the asymptotic scaling properties of the deconfinement transition in the $S U(2)$ pure gauge theory. In this letter we extend the analysis to the deconfinement transition in $S U(3)$ theory. Our main purpose is a better understanding of the effect of the perturbative improvement in the context of 4 dimensional gauge theories. In this respect the deconfinement temperature $T_{c}$ is an observable particularly well suited to give unambiguous answers. As early noticed [3 5] it can be accurately measured; being a bulk effect it has a slow volume dependence and most importantly it is not affected by perturbative contributions which violate the scaling. We determine the critical coupling of the decon-

${ }^{1}$ Partially supported by M.U.R.S.T., Italy

2 Partially supported by I.N.F.N., Italy 
finement transition in lattices with $N_{\tau}=3,4,5,6$, and study the pattern of asymptotic scaling violation of $T_{c}$, in comparison with the existing Wilson action simulations (for good reviews see [6,7] and references therein), both in the "bare" scheme and in the effective schemes based on the expectation values of plaquettes. In the bare scheme we find that the two lattice formulations give quite similar results, taking into account the redefinition of the $\Lambda$ scale, which corresponds to the perturbative prediction whitin $10 \%$. This is a confirmation that the Wilson and Symanzik actions are in the same universality class, and that the $\beta$ function is not affected, in the "bare" scheme, by the irrelevant operators connecting the two lattice formulations: on the other hand the results show further that with the currently accessible lattices the two-loop perturbative approximation is not sufficient to model the renormalization group flow in the scaling region. In sec. (2) we give some details on our simulation and on the numerical results, discussing the consequences of an effective coupling parameterization. In sec. (3) we discuss a test of our simulation, aimed to verify the first order nature of the deconfinement transition through a F.S.S. analysis on $N_{\tau}=3$ lattices.

\section{The simulation}

We have considered the $S U(3)$ gauge theory on asymmetric lattices using the lowest order tree improved Symanzik action

$$
S_{\mathrm{I}}=\beta \sum \operatorname{Re} \operatorname{Tr}\left(\frac{5}{3} U_{1 \times 1}-\frac{1}{12} U_{1 \times 2}\right),
$$

defined in order to remove $O\left(a^{2}\right)$ scaling violations in the Callan-Symanzik equation for physical quantities measured on the lattice: we refer to our preceding work [1] and references therein for a more detailed discussion of the improvement strategies and of the results existing in literature. We have performed simulations on lattices $N_{\tau} \times N_{\sigma}^{3}=3 \times 12^{3}, 4 \times 16^{3}, 5 \times 20^{3}, 6 \times 24^{3}$; the update is done with a combination of Heat-Bath and of overrelaxation sweeps applied to the $S U(2)$ subgroups [8]. All the data have been obtained on the APE machines installed in Pisa, including a 128 processors machine with a peak speed of 6 GigaFlops. We have measured the order parameter of the deconfining transition, the spatial average of the Polyakov line

$$
P=N_{\sigma}^{-3} \sum_{\vec{x}} \operatorname{Tr} \prod_{t=1}^{N_{t}} U_{4}(\vec{x}, t)
$$


As usual (see for example [9]) to consider the projection of this quantity on the nearest $Z(3)$ axis,

$$
\Omega=\left\{\begin{array}{ll}
\operatorname{Re} P & \arg P \in\left(-\frac{\pi}{3}, \frac{\pi}{3}\right) \\
\operatorname{Re} P \exp (-2 i \pi / 3) & \arg P \in\left(\frac{\pi}{3}, \pi\right) \\
\operatorname{Re} P \exp (2 i \pi / 3) & \arg P \in\left(-\pi,-\frac{\pi}{3}\right)
\end{array},\right.
$$

in order to avoid the vanishing of the order parameter, on finite lattices, induced by the tunnelling between broken phases. We locate the deconfinement transition by studying the Polyakov susceptibility

$$
\chi(\Omega)=N_{\sigma}^{3}\left\langle(\Omega)^{2}-\langle\Omega\rangle^{2}\right\rangle
$$

which exhibits a clear peak structure, and the specific heat of the lattice action. The density of states method [10] has been used to extrapolate data coming from runs at different $\beta$ values and to combine them in a single curve: the data autocorrelation has been taken in account using a standard binning procedure, varying the bin size and testing the existence of a plateau in the variance of the binned quantities. In order to reduce the bias induced by the finite sample size we have used the techniques discussed in [11]: "standard" and "jackknife" estimators have been defined, combining them with coefficients appropriate to cancel the $\frac{1}{N}$ term in the bias. In fig. (1) is given an example of the resummation of the Polyakov susceptibility around the deconfining transition on the $N_{\tau}=3$ lattice, where the actual simulation values are addressed by a black square. A more extended discussion of the numerical simulation will be given elsewhere. In tab. (1) we report the values of the (pseudo) critical coupling on the different lattices, obtained by fitting the peaks with a gaussian curve, and the corresponding critical temperature in units of $\Lambda_{I}$, obtained by means of the two loop R.G. formula

$$
\frac{T_{c}}{\Lambda_{I}}=\frac{1}{a N_{\tau} \Lambda_{I}}=\frac{1}{N_{\tau}}\left(\frac{\beta}{2 C_{a} b_{0}}\right)^{-b_{1} / 2 b_{0}^{2}} \exp \left(\frac{\beta}{4 C_{a} b_{0}}\right) .
$$

They are also reported the corresponding quantities obtained in simulations using the Wilson action [3, 5, 12, In the last column the corresponding ratio of the $\Lambda$ parameters in the improved and standard formulation is listed, showing at $N_{\tau}=6$ a $10 \%$ discrepancy from the perturbative prediction [13]

$$
\frac{\Lambda_{I}}{\Lambda_{W}}=5.29210(1)
$$

It is instructive to compare different actions and schemes by normalizing the results in order to have the values at smaller a coincide, as in fig. (2). It is quite apparent that the asymptotic scaling violations for the Symanzik and Wilson action, in the "bare" scheme, are similar, as already noted in the

FIG. 2 


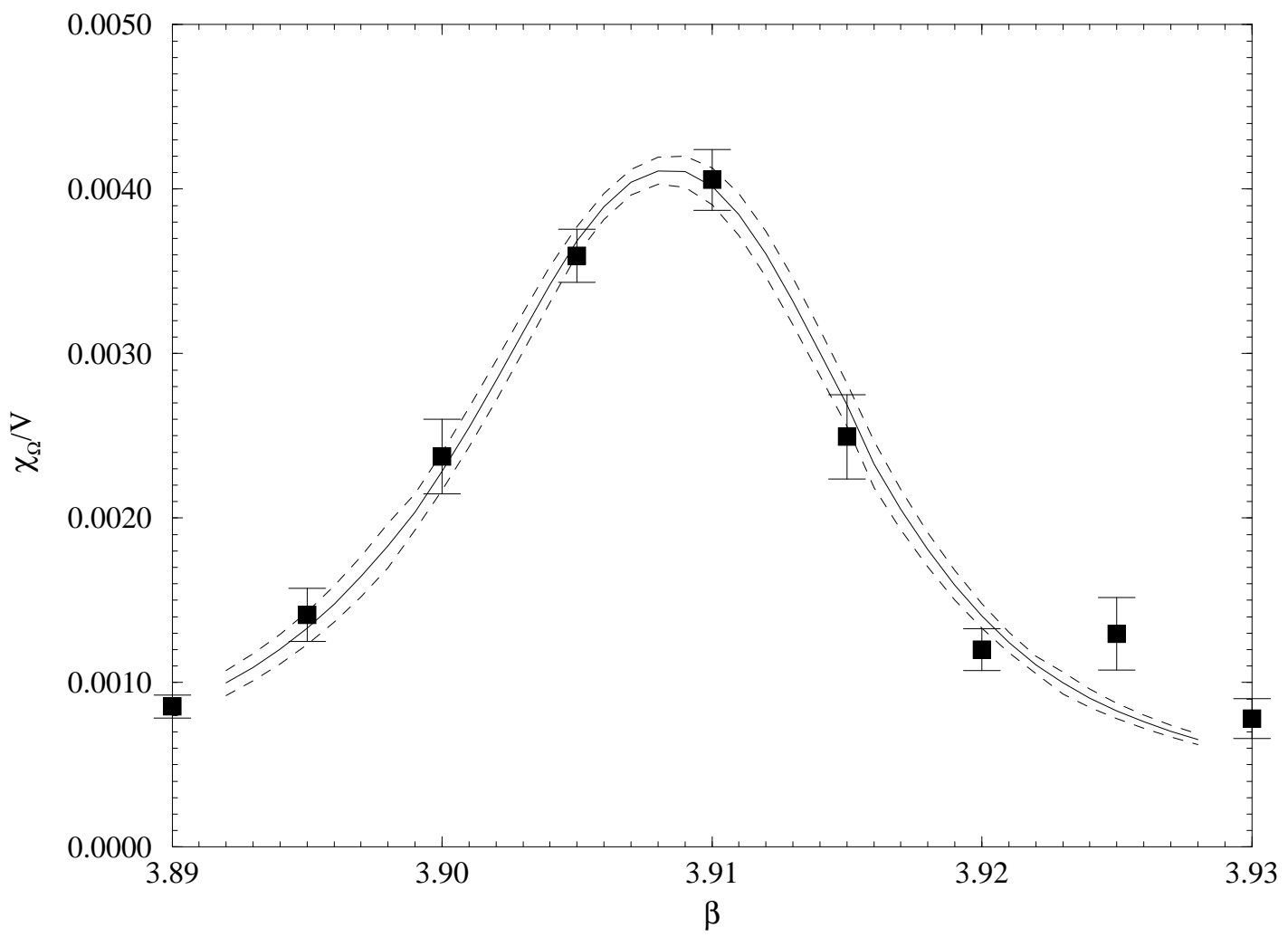

Fig. 1. Susceptibility of the Polyakov line on a $3 \times 12$ lattice

Table 1

$\beta_{c}$ values on different lattices

\begin{tabular}{c|c|c|c|c|c}
\hline$N_{\tau}$ & $\beta_{c}$ (Improved) & $T_{c} / \Lambda_{I}$ & $\beta_{c}$ (Wilson) & $T_{c} / \Lambda_{W}$ & $\Lambda_{I} / \Lambda_{W}$ \\
3 & $3.90812(7)$ & $13.937(2)$ & $5.55(1)$ & $85.70(96)$ & $6.39(7)$ \\
4 & $4.07252(13)$ & $12.505(2)$ & $5.6925(2)$ & $75.41(2)$ & $6.030(2)$ \\
5 & $4.19963(14)$ & $11.497(2)$ & $5.7933(3)$ & $68.5(1)$ & $5.95(1)$ \\
6 & $4.31466(24)$ & $10.870(3)$ & $5.8941(5)$ & $63.05(4)$ & $5.800(5)$
\end{tabular}

$S U(2)$ simulation [1]]. In the same figure we show the same data parameterized in terms of the expectation values of plaquettes, in the manner of Parisi [14]: to define effective couplings, the perturbative predictions on the $1 \times 1$ and $1 \times 2$ plaquettes [13] have been used, together with the results of simulations on large symmetric lattices. As already noted [7] for the results obtained with the Wilson action, this parameterization shows an apparent asymptotic scaling already for $N_{\tau} \geq 4$; our simulation with the Symanzik tree improved action shows an apparent scaling already for $N_{\tau}=3$. 


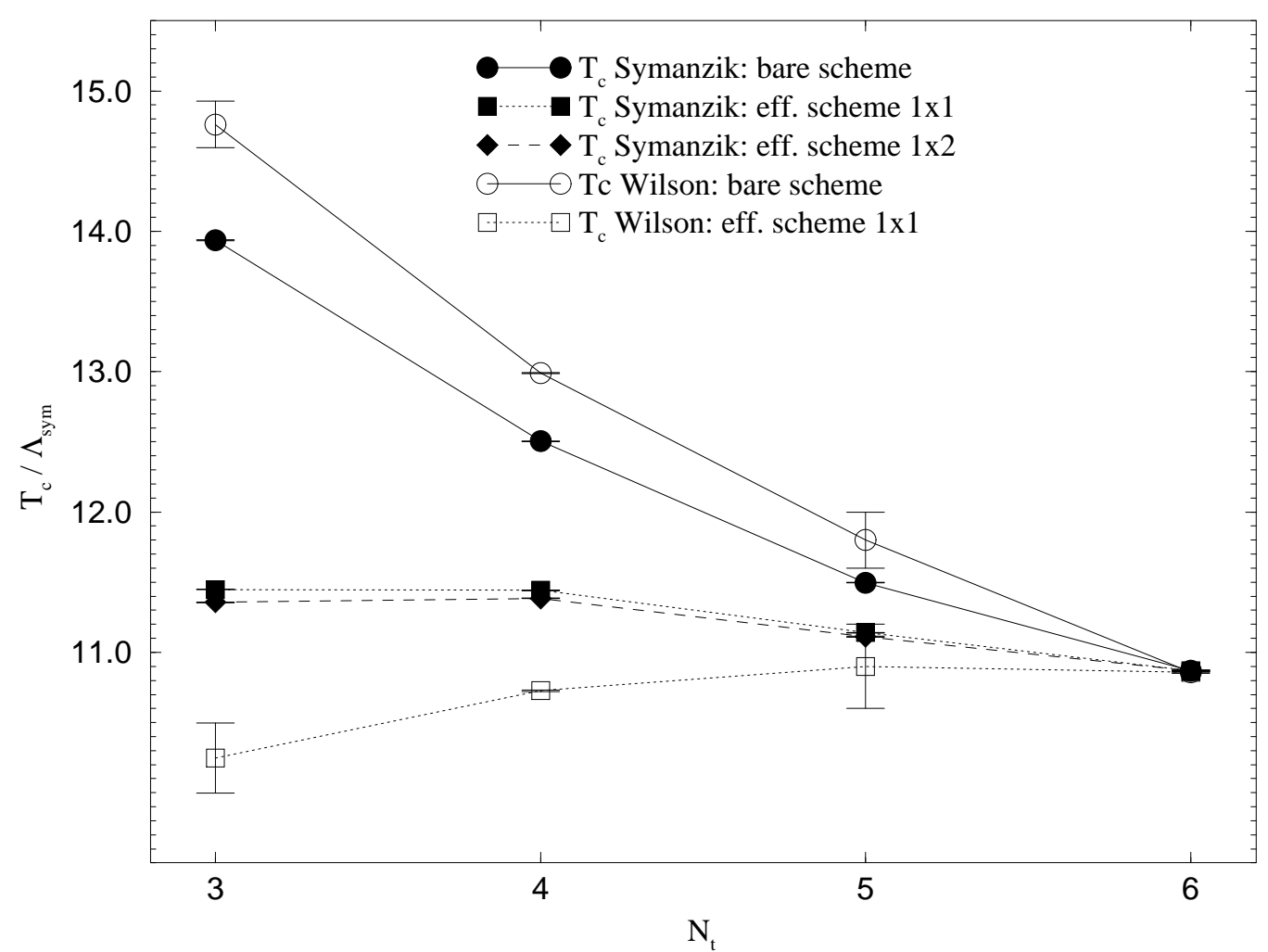

Fig. 2. Asymptotic scaling figure for the critical temperature

We recall that the Symanzik approach is based on perturbation theory: hence the fact that the improvement is apparent when an effective coupling is used seems a confirmation of the work of Lepage and Mackenzie [15], which asserts that the bare coupling is a bad expansion parameter, and that the perturbative series, when properly reorganized, work well already on the accessible lattices. In other words the effect of the Symanzik improvement is "buried" by the large renormalizations induced by the lattice formulation: the improvement shows up when a scheme is chosen which minimizes these finite corrections.

\section{Finite size study for $N_{\tau}=3$}

In order to control at least in part the systematic error connected to the finite size of the lattices, we have performed for $N_{\tau}=3$ an additional set of simulations with $N_{\sigma}=6,7,8,9,10,11$, to test the dependence of the critical coupling on the volume of the system, and to check the expected scaling relations for a first order phase transition. We report in tab. (2) the values of the position of the peak of the susceptibility of the Polyakov line and its height, resulting from a gaussian fit with a self consistent data windowing around the peak. In fig. (3) we compare the results for the Polyakov line susceptibility: we

FIG. 3 


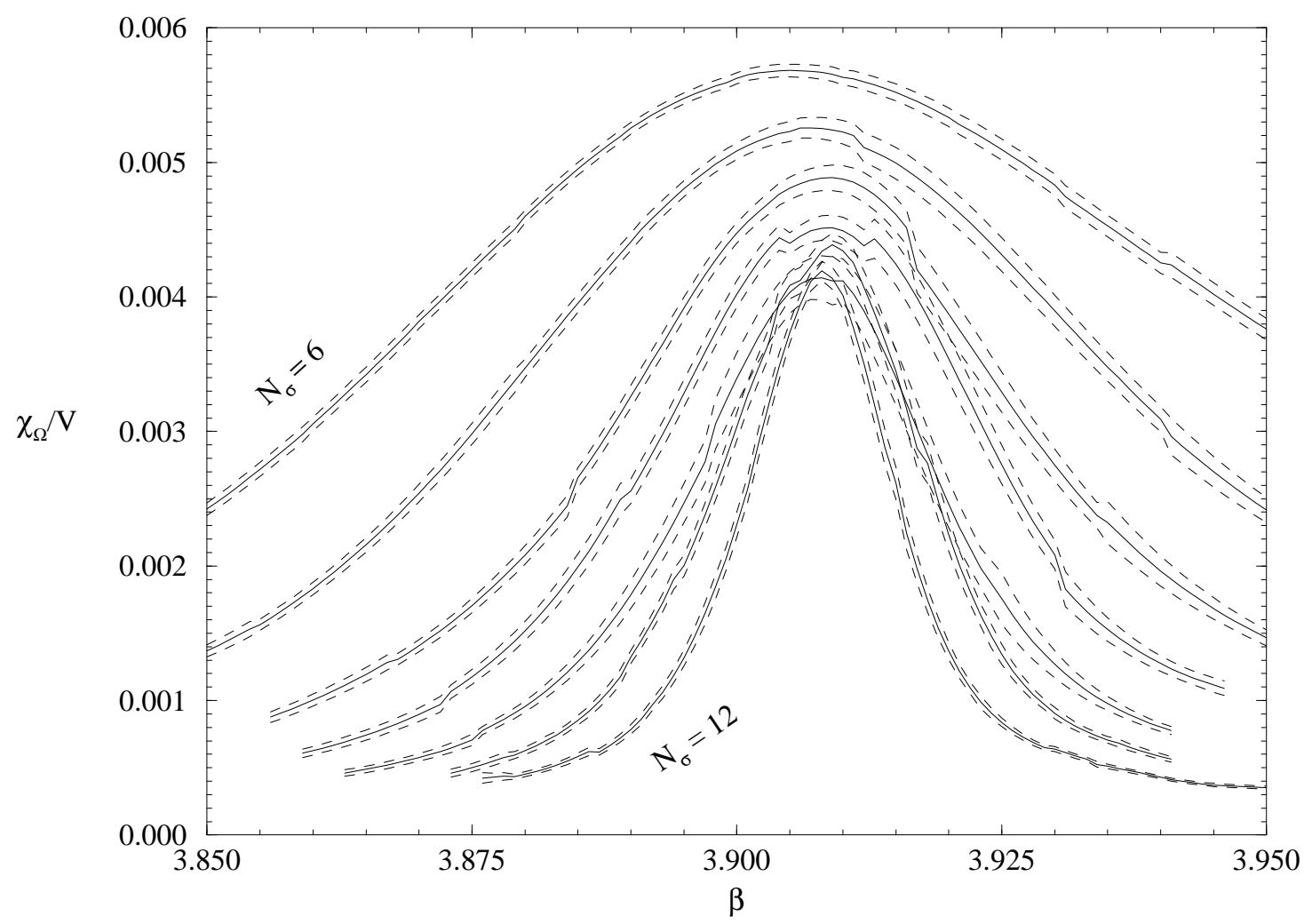

Fig. 3. Polyakov susceptibility for $N_{\tau}=3, N_{\sigma}=6, \ldots 12$

Table 2

Results of the finite size study on the $N_{\tau}=3$ lattices.

\begin{tabular}{c|c|c}
\hline$N_{\sigma}$ & $\beta_{c}^{\max }$ & $\chi(\Omega)^{\max } / N_{\sigma}^{3}$ \\
\hline 6 & $3.90588(34)$ & $0.00568(5)$ \\
7 & $3.90525(53)$ & $0.00514(12)$ \\
8 & $3.90825(46)$ & $0.00505(12)$ \\
9 & $3.90859(24)$ & $0.00450(12)$ \\
10 & $3.90765(5)$ & $0.00415(16)$ \\
11 & $3.90853(8)$ & $0.00436(9)$ \\
12 & $3.90812(7)$ & $0.00416(7)$
\end{tabular}

note that the scaling of the peak is compatible with the characteristic behavior in presence of a first order phase transition

$$
\chi(\Omega) \simeq N_{\sigma}^{3} .
$$


Most importantly for our purposes, we note that the shift of the pseudocritical coupling corresponding to the peak of the transition is modest, giving confidence in the values and the errors quoted in tab. (1) for the lattices with $N_{\tau}>3$.

\section{Conclusions}

In this work we have performed a study of the deconfinement phase transition in the pure gauge $S U(3)$ theory, using the Symanzik tree improved action and determining the critical couplings on lattices with $N_{\tau}=3,4,5,6$. The main conclusion is that, as in the case of the deconfinement transition in $S U(2)$ theory [1], the same pattern of asymptotic scaling violation is observed. The asymptotic scaling violations are much reduced if the two loop perturbative expression is used in conjunction with effective couplings, based on UV dominated quantities like the plaquette expectation values, and comparison with the results of simulations with the Wilson action, in this "effective" schemes, shows a slighlty precocious scaling, which is tempting to interpret as an effect of the improvement. Although we do not have under control the systematical errors due to the finite volume, the analysis of the finite size behavior in the $N_{\tau}=3$ lattices makes us confident that the quoted results are not much affected by the finite size shift of the transition.

\section{Acknowledgement}

We wish to thank R. Tripiccione for many useful discussions and for a careful reading of the manuscript. We also acknowledge the support of the APE group, particularly S. Cabasino and G. M. Todesco.

\section{References}

[1] G. Cella, G. Curci, R. Tripiccione and A. Viceré, Phys. Rev. D49 (1994) 511.

[2] K. Symanzik, Nucl. Phys. B226 (1983) 187; Nucl. Phys. B226 (1983) 205; in New developments in gauge theories, ed. G. 't Hooft et al. (Plenum, New York, 1980); in Lecture Notes in Physics 153, ed. R. Schrader et al. (Springer, Berlin, 1982); in Non-perturbative field theory and QCD, ed. R. Jengo et al. (World Scientific, Singapore, 1983).

[3] F. Karsch and R. Petronzio, Phys. Lett. B139 (1984) 403. 
[4] Gottlieb et al. Phys. Rev. Lett. 55 (1985) 1958.

[5] Kennedy et al. Phys. Rev. Lett. 54 (1985) 87.

[6] B. Petersson, in Nucl. Phys. B(Proc. Suppl.)30 (1993) 66.

[7] J. Fingberg, U. Heller and F. Karsch, Nucl. Phys. B392 (1993) 493.

[8] N. Cabibbo and E. Marinari, Phys. Lett. B119 (1982) 387.

[9] M. Fukugita, M. Okawa and A. Ukawa, Nucl. Phys. B337 (1990) 181.

[10] A. M. Ferrenberg and R. H. Swendsen, Phys. Rev. Lett. 61 (1988) 2635; Phys. Rev. Lett. 63 (1989) 1195.

[11] B. A. Berg. Comput. Phys. Commun. 69 (1991) 7. N. A. Alves, B. A. Berg, S. Sanielevici, Nucl. Phys. B376 (1993) 218.

[12] Iwasaki et al. Phys. Rev. Lett. 67 (1991) 3343.

[13] P. Weisz and R. Wohlert, Nucl. Phys. B236 (1984) 397; Nucl. Phys. B247 (1984) 544 .

[14] G. Parisi, Proceedings of the XXth Conf. on High energy physics (Madison, WI 1980). G. Martinelli, G. Parisi and R. Petronzio, Phys. Lett. B100 (1981) 485.

[15] G. P. Lepage and P. B. Mackenzie, Phys. Rev. D48 (1993) 2250. 